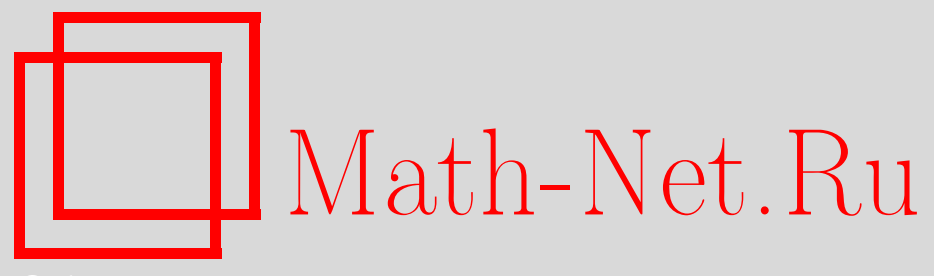

В. Г. Кротов, М. А. Прохорович, Скорость сходимости средних Стеклова на метрических пространствах с мерой и размерность Хаусдорфа, Матем. заметки, 2011, том 89, выпуск 1, 145-148

DOI: https://doi.org/10.4213/mzm8927

Использование Общероссийского математического портала Math-Net.Ru подразумевает, что вы прочитали и согласны с пользовательским соглашением http://www . mathnet.ru/rus/agreement

Параметры загрузки:

IP: 34.229 .108 .108

26 апреля 2023 г., $17: 48: 41$ 


\section{Скорость сходимости средних Стеклова на метрических пространствах с мерой и размерность Хаусдорфа}

\section{В. Г. Кротов, М. А. Прохорович}

Пусть $(X, d, \mu)$ - метрическое пространство с метрикой $d$ и регулярной борелевской мерой $\mu$. Введем обозначения

$$
B(x, r)=\{y \in X: d(x, y)<r\}
$$

- шар с центром в точке $x \in X$ радиуса $r>0, r_{B}$ - радиус шара $B, \lambda B$ - шар радиуса $\lambda r_{B}$, концентрический с $B$,

$$
f_{B}=f_{B} f d \mu=\frac{1}{\mu(B)} \int_{B} f d \mu
$$

- среднее значение функции $f \in L^{1}(B)$ по шару $B \subset X$ (средние Стеклова). Через $L^{p}=L^{p}(X), 1 \leqslant p<\infty$, обозначаем обычные лебеговы пространства, порожденные мерой $\mu$.

Мы будем иметь дело со свойствами функций, которые зависят от изменения их значений на множестве меры нуль, поэтому условимся, что значение локально суммируемой функции в каждой точке определяется равенством $f(x)=\varlimsup_{\lim }^{r \rightarrow+0} f_{B(x, r)}$.

Ниже считаем выполненным условие удвоения $\mu(B(x, 2 r)) \leqslant c \mu(B(x, r)){ }^{1}$ тогда при некотором $\gamma>0$

$$
\mu\{B(x, R)\} \leqslant c\left(\frac{R}{r}\right)^{\gamma} \mu(B(x, r)), \quad x \in X, \quad 0<r \leqslant R .
$$

Параметр $\gamma$ важен в дальнейшем - он выполняет роль размерности $X$.

Рассмотрим максимальные функции

$$
\mathscr{S}_{\alpha} f(x)=\sup _{B \ni x} r_{B}^{-\alpha} f_{B}\left|f(y)-f_{B}\right| d \mu(y)
$$

где точная верхняя грань берется по всем шарам $B, r_{B} \in(0,1)$, содержащим точку $x \in X$. $\mathrm{C}$ помощью этих максимальных функций определим классы

$$
C_{\alpha}^{p}=C_{\alpha}^{p}(X)=\left\{f \in L^{p}:\|f\|_{C_{\alpha}^{p}}=\|f\|_{L^{p}}+\left\|\mathscr{S}_{\alpha} f\right\|_{L^{p}}<\infty\right\} .
$$

Для $\mathbb{R}^{n}$ это определение восходит к работам [1], [2] (см. подробнее [3]), а в общем случае оно было введено в [4], [5]. Класс $C_{1}^{p}(X)$ совпадает с классом Хайлаша-Соболева [6] (см. также [5], [7]), а при $X=\mathbb{R}^{n}-$ с классическим пространством Соболева $W_{1}^{p}\left(\mathbb{R}^{n}\right)$ [1], [6].

Напомним определение хаусдорфовых $s$-вместимости $\mathbb{H}_{\infty}^{s}(E)$ и размерности $\operatorname{dim}_{\mathbb{H}}(E)$ множества $E \subset X$ :

$$
\mathbb{H}_{\infty}^{s}(E)=\inf \left\{\sum_{i=1}^{\infty} r_{i}^{s}: E \subset \bigcup_{i=1}^{\infty} B\left(x_{i}, r_{i}\right)\right\}, \quad \operatorname{dim}_{\mathbb{H}}(E)=\inf \left\{s: \mathbb{H}_{\infty}^{s}(E)=0\right\}
$$

В таких терминах в [8]-[10] изучался вопрос о массивности множества точек Лебега для функций из классов $C_{\alpha}^{p}$. Приведем итоговый результат.

\footnotetext{
1 Здесь и всюду дальше через $c$ обозначаем различные положительные постоянные, значения которых не играют роли.
}

(C) В. Г. Кротов, М. А. Прохорович, 2011 
Tеорема 1. Пусть $\alpha>0,1<p<\gamma / \alpha$. Тогда для любой функиии $f \in C_{\alpha}^{p}$ существует множество $E \subset X$ такое, что $\operatorname{dim}_{\mathbb{H}}(E) \leqslant \gamma-\alpha p$ и для любого $x \in X \backslash E$ существует предел

$$
\lim _{r \rightarrow+0} f_{B(x, r)} f d \mu=f^{*}(x),
$$

более того,

$$
\lim _{r \rightarrow+0} f_{B(x, r)}\left|f-f^{*}(x)\right|^{q} d \mu=0 \quad n p u \quad \frac{1}{q}=\frac{1}{p}-\frac{\alpha}{\gamma} .
$$

История подобных результатов на $\mathbb{R}^{n}$ достаточно подробно изложена в $[11 ;$ гл. 6.2]. Для $\alpha=1$ утверждение теоремы 1 было частично получено в [8; теорема 4.4]. Случай $0<\alpha \leqslant 1$ был рассмотрен в [9; теорема 1], а в [10; теорема 1] было снято ограничение $\alpha \leqslant 1$. Вопрос о том, можно ли в теореме 1 дополнительно утверждать, что $\mathbb{H}_{\infty}^{\gamma-\alpha p}(E)=0$, остается открытым.

В нашей работе мы дадим решение аналогичной задачи для скорости сходимости средних Стеклова для функций из классов $C_{\alpha}^{p}$.

Теорема 2. Пусть $\alpha>0,1<p<\gamma / \alpha, 0<\beta<\alpha$. Тогда для любой функции $f \in C_{\alpha}^{p}$ существует множество $E \subset X$ такое, что

1) $\mathbb{H}_{\infty}^{\gamma-(\alpha-\beta) p}(E)=0$, в частности $\operatorname{dim}_{\mathbb{H}}(E) \leqslant \gamma-(\alpha-\beta) p$;

2) для всех $x \in X \backslash E$

$$
\lim _{r \rightarrow+0} r^{-\beta}\left(f_{B(x, r)}|f-f(x)|^{q} d \mu\right)^{1 / q}=0 \quad \text { npu } \quad \frac{1}{q}=\frac{1}{p}-\frac{\alpha}{\gamma} .
$$

При $\alpha \leqslant 1$ утверждение теоремы 1 можно вывести из результатов [12; следствие 6] и [9; теорема 2]. Однако условие $\alpha \leqslant 1$ существенно сужает множество возможных ситуаций для некоторых $X$ классы Гёльдера $H^{\alpha} \subset C_{\alpha}^{p}$ могут быть нетривиальны при некоторых значениях $\alpha>1$ (см., например, [13; пример 1]).

Для доказательства теоремы 2 нам понадобится ряд вспомогательных фактов. Прежде всего отметим, что для любого шара $B \subset X$ и $p>0$

$$
f_{B}\left|f-f_{B}\right| d \mu \leqslant r_{B}^{\alpha}\left(f_{B}\left[\mathscr{S}_{\alpha} f\right]^{p} d \mu\right)^{1 / p} .
$$

Это следует из усреднения по $y \in B$ очевидного неравенства

$$
f_{B}\left|f-f_{B}\right| d \mu \leqslant r_{B}^{\alpha} \mathscr{S}_{\alpha} f(y), \quad y \in B .
$$

Лемма 1. Если $0<\delta<\gamma u g \in L^{1}$, mo

$$
\mathbb{H}_{\infty}^{\gamma-\delta}(E)=0, \quad \text { əде } \quad E=\left\{x \in X: \varlimsup_{r \rightarrow+0} r^{\delta} f_{B(x, r)}|g| d \mu>0\right\} .
$$

Лемма 2. Если $f \in L_{\mathrm{loc}}^{1}, p>0,0<\alpha<\gamma / p$, то для любого шара $B$

$$
\left(f_{B}\left|f-f_{B}\right|^{q} d \mu\right)^{1 / q} \leqslant c r_{B}^{\alpha}\left(f_{2 B}\left[\mathscr{S}_{\alpha} f\right]^{p} d \mu\right)^{1 / p}, \quad \frac{1}{q}=\frac{1}{p}-\frac{\alpha}{\gamma} .
$$

Лемма 1 имеется в работе второго автора [10; лемма 3], а лемма 2 является частным случаем результатов работы [14; теорема 1]. 
ДокаЗАТЕЛЬСтво теоремы 2. Пусть $E_{1}$ - дополнение ко множеству точек $x \in X$, для которых выполнено соотношение

$$
\lim _{r \rightarrow+0}\left[f(x)-f_{B(x, r)}\right]=0 ;
$$

тогда по теореме 1

$$
\mathbb{H}_{\infty}^{\gamma-(\alpha-\beta) p}\left(E_{1}\right)=0 .
$$

Пусть $x \in X \backslash E_{1}, r \in(0,1)$ и $B_{j}=B\left(x, 2^{-j} r\right)$ для $j \in \mathbb{N}$. Тогда

$$
\left|f(x)-f_{B(x, r)}\right| \leqslant \sum_{j=0}^{\infty}\left|f_{B\left(x, 2^{-j-1} r\right)}-f_{B\left(x, 2^{-j} r\right)}\right| .
$$

Отсюда в силу условия удвоения (1) и неравенства (3) получаем

$$
\begin{aligned}
& r^{-\beta}\left|f(x)-f_{B(x, r)}\right| \\
& \quad \leqslant r^{-\beta} \sum_{j=0}^{\infty} \frac{\mu\left(B_{j}\right)}{\mu\left(B_{j+1}\right)} f_{B_{j}}\left|f-f_{B_{j}}\right| d \mu \leqslant c \sum_{j=0}^{\infty} 2^{-j \beta}\left(r_{B_{j}}^{(\alpha-\beta) p} f_{B_{j}}\left[\mathscr{S}_{\alpha} f\right]^{p} d \mu\right)^{1 / p} \\
& \quad \leqslant c \sup _{t<r}\left(t^{(\alpha-\beta) p} f_{B(x, t)}\left[\mathscr{S}_{\alpha} f\right]^{p} d \mu\right)^{1 / p},
\end{aligned}
$$

и правая часть сходится к нулю при $r \rightarrow+0$, если

$$
\lim _{r \rightarrow+0} r^{(\alpha-\beta) p} f_{B(x, r)}\left[\mathscr{S}_{\alpha} f\right]^{p} d \mu=0 .
$$

В силу леммы 1 , примененной к функции $g=\left[\mathscr{S}_{\alpha} f\right]^{p}$ и $\delta=(\alpha-\beta) p$,

$$
\mathbb{H}_{\infty}^{\gamma-(\alpha-\beta) p}\left(E_{2}\right)=0, \quad E_{2}=\left\{x \in X: \varlimsup_{r \rightarrow+0} r^{(\alpha-\beta) p} f_{B(x, r)}\left[\mathscr{S}_{\alpha} f\right]^{p} d \mu>0\right\} .
$$

Итак, на дополнении ко множеству $E=E_{1} \cup E_{2}$ выполнено соотношение (5), а по лемме 2

$$
r^{-\beta}\left(f_{B(x, r)}\left|f-f_{B(x, r)}\right|^{q} d \mu\right)^{1 / q} \leqslant c\left(r^{(\alpha-\beta) p} f_{B(x, 2 r)}\left[\mathscr{S}_{\alpha} f\right]^{p} d \mu\right)^{1 / p},
$$

и для $x \in X \backslash E_{2}$ правая часть сходится к нулю при $r \rightarrow+0$. Следовательно, для любого $x \in X \backslash E$

$$
\begin{aligned}
\varlimsup_{r \rightarrow+0} & r^{-\beta}\left(f_{B(x, r)}|f-f(x)|^{q} d \mu\right)^{1 / q} \\
& \leqslant \lim _{r \rightarrow+0} r^{-\beta}\left|f(x)-f_{B(x, r)}\right|+\lim _{r \rightarrow+0} r^{-\beta}\left(f_{B(x, r)}\left|f-f_{B(x, r)}\right|^{q} d \mu\right)^{1 / q}=0 .
\end{aligned}
$$

Осталось заметить, что $\mathbb{H}_{\infty}^{\gamma-(\alpha-\beta) p}(E)=0$ в силу (6) и $(7)$.

\section{СПИСОК ЦИТИРОВАННОЙ ЛИТЕРАТУРЫ}

[1] A. P. Calderón, Studia Math., 44 (1972), 563-582. [2] A. P. Calderón, R. Scott, Studia Math., 62:1 (1978), 75-92. [3] R. A. DeVore, R. C. Sharpley, Maximal Functions Measuring Smoothness, Mem. Amer. Math. Soc., 47, № 293, 1984. [4] J. Hu, J. Math. Anal. Appl., 280:1 (2003), 91-101. [5] D. Yang, Sci. China Ser. A, 46:5 (2003), 675-689. [6] P. Hajłasz, Potential Anal., 5:4 (1996), 403-415. [7] И. А. Иванишко, Матем. заметки, 77:6 (2005), 937-941. 
[8] P. Hajłasz, J. Kinnunen, Rev. Mat. Iberoamericana, 14:3 (1998), 601-622. [9] M. A. Пpoxoрович, Матем. заметки, 82:1 (2007), 99-107. [10] М. А. Прохорович, Матем. заметки, 85:4 (2009), 616-621. [11] D. R. Adams, L. I. Hedberg, Function Spaces and Potential Theory, Grundlehren Math. Wiss., 314, Springer-Verlag, Berlin, 1996. [12] В. Г. Кротов, Изв. НАН Армении. Сер. матем., 41:2 (2006), 25-42. [13] A. Jonsson, J. Math. Anal. Appl., 290:1 (2004), 86-104. [14] И. А. Иванишко, В. Г. Кротов, Тр. Ин-та матем. НАН Беларуси, 14:1 (2006), 51-61.

\section{В. Г. Кротов}

Поступило

Белорусский государственный университет, г. Минск

18.05 .2010

E-mail: krotov@bsu.by

\section{М. А. Прохорович}

Белорусский государственный университет, г. Минск

E-mail: prokhorovich@bsu.by 